\title{
Archives of Pediatric Neurosurgery: the challenges of implementing an online journal
}

\author{
Ricardo Santos de Oliveira, PhD
}

Received: 10 July 2019 / Accepted: 11 July 2019

The Brazilian Society for Pediatric Neurosurgery has become mature since its foundation in 1999. The Brazilian pediatric neurosurgeons have been recognized around the world and have been playing an important role in the dissemination of high level knowledge.

Medical journals will continue as a main vehicle of scientific information for years to come, particularly where access to more efficient instruments is relatively limited. With the development of electronic publishing, many medical journals now have web sites on the Internet, and some journals have transitioned to online-only publications.

The quality of medical journals depends on several factors involving 3 groups of people; namely: the authors, the reviewers and the editors. Archives of Pediatric Neurosurgery (APN) formulated its essential requirements for the manuscripts submitted for possible publication. The reviewers' role is a keystone in maintaining the quality of a medical jornal. A reviewer is required to address several important aspects of the paper under review and to resend his opinion thereon with his recommendation concerning the acceptability of the paper or otherwise. The editorial management is a crucial part of the publishing

Division of Pediatric Neurosurgery, Department of Surgery and Anatomy, Ribeirao Preto Medical School, University of Sao Paulo, Brazil

To whom correspondence should be addressed: Ricardo Santos de Oliveira, MD, PhD [E-mail: rsoliveira30@gmail.com] process. The editors begin action with the receipt of the manuscript, direct the various steps of evaluation, correction and re-submission, until an editorial decision is made to accept the paper as is, accept it after modification or reject it. Editors will then make necessary text and layout editing. Due consideration is given to the statistical, multilingual and ethical aspects as well as to the overall uniformity of the terminology, nomenclatures and style throughout the volume as a whole.

Each issue contains authoritative review articles, original articles, technical notes commentaries on current and previously published papers, book reviews, and letters to the Editor. Original manuscripts and Brief Communications are given priority for publication. Case reports are well accepted, although preference is for those that might generate discussion or provide an update on rare subjects.

When writing your case report please consider that the mission for APN "... is to use clinical case reports to disseminate the best clinical practice, to examine important common as well as uncommon clinical scenarios, and to illustrate and inform the use of important clinical guidelines and systematic reviews."

Archives of Pediatric Neurosurgery represents the birth of a modern journal taking the Brazilian Pediatric Neurosurgery for another level.

Let's grow together!

Key words: Pediatric neurosurgery, Online, Medical

journal

Journal homepage: www.sbnped.com.br 